\title{
Renal cortical stiffness is markedly increased in pre-diabetes mellitus and associated with albuminuria
}

\author{
Hilmi Erdem Sumbul${ }^{1}$, MD, Ayse Selcan $\underline{K o c}^{2}$, MD, Erdinç Gülümsek ${ }^{1}$, MD
}

INTRODUCTION We aimed to investigate changes in renal cortical stiffness (CS) in Type 2 diabetes mellitus (DM) and pre-DM patients compared to subjects with normal glucose metabolism (NGM), as well as the usefulness of renal CS to determine the presence of nephropathy.

METHODS This study included 125 individuals with NGM, pre-DM and Type 2 DM. Routine laboratory data was obtained, and micro- and macrovascular involvement were investigated. Urinary albumin-creatinine ratio (UACR) was measured for urinary albuminuria detection. In addition to routine renal ultrasonography, CS was measured using renal elastography. RESULTS Among the included patients, 42, 40 and 43 patients had NGM, pre-DM and Type 2 DM, respectively, with prevalence of nephropathy of $5 \%, 15 \%$ and $33 \%$, respectively. Carotid and aortic intima-media thickness (IMT), renal width, and CS were found to be higher in the pre-DM and Type 2 DM groups than the NGM group. Aortic IMT, renal width and UACR levels were independently associated with CS. Patients with nephropathy were found to have a higher CS value than those without nephropathy $(8.72 \pm 1.67 \mathrm{kPa}$ vs. $10.60 \pm 1.74 \mathrm{kPa}, \mathrm{p}=0.001)$. In receiver operating characteristic curve analysis, when the cut-off value for CS was taken as $9.2 \mathrm{kPa}$, renal CS predicted the possibility of nephropathy with $78.9 \%$ sensitivity and $71.4 \%$ specificity.

CONCLUSION CS values are significantly higher in patients with impaired glucose metabolism. We recommend CS measurement as part of routine screening of nephropathy in patients with pre-DM and newly diagnosed Type 2 DM.

Keywords: albuminuria, cortical stiffness, diabetes mellitus, microvascular complications, pre-DM

\section{INTRODUCTION}

Diabetes mellitus (DM) is a chronic, progressive disease that causes long-term microvascular and macrovascular complications in many organs, including the eye, kidney, heart and peripheral nerves. Organ involvement is the most common cause of increased DM-related mortality and morbidity. Impaired fasting glucose and impaired glucose tolerance are two known prediabetic glucose metabolic disorders associated with microvascular and macrovascular complications. ${ }^{(1-11)}$ Epidemiologic studies have reported a $14 \%$ prevalence of pre-DM in populations over the age of $45,{ }^{(12,13)}$ suggesting that the lifelong risk of developing DM in these individuals may be $48 \%-74 \%$. $^{(12,13)}$ The prevalence of pre-DM and DM is estimated to be 592 million and 471 million people in 2035, respectively. ${ }^{(14)}$ Thus, precautions taken for early diagnosis and treatment of both clinical conditions may prevent or delay the development of microvascular and macrovascular complications.

Pre-DM and newly diagnosed Type 2 DM patients have a significant presence of kidney disease, suggesting that they may develop nephropathy before DM develops. ${ }^{(15-17)}$ The Rotterdam study ${ }^{(13)}$ and Melsom et al ${ }^{(12)}$ reported that early treatment of preDM patients could prevent the onset of diabetic kidney disease (DKD). For this reason, it is important to diagnose this disease early in the course of pre-DM and before the development of nephropathy. Urinary albumin-creatinine ratio (UACR) can be used to detect early development of nephropathy, but there is still no objective parameter predicting the development of nephropathy. ${ }^{(12,18)}$ Studies have shown that changes at the cellular level occur before the diagnosis of nephropathy-related abnormalities. ${ }^{(19)}$

Shear wave elastography (SWE) has recently become a commonly used technique and has begun to be used in patients with DM. It has been shown that patients with Type 2 DM have an increase in renal cortical stiffness (CS) that is observed on SWE. ${ }^{(20-22)}$ However, to our knowledge, there is no information in the literature regarding changes in CS in patients with pre-DM and newly diagnosed Type 2 DM. We hypothesise that CS, as determined by SWE, may indicate early changes at the cellular level prior to the onset of nephropathy in patients with pre-DM and newly diagnosed Type 2 DM. In this study, we aimed to investigate changes in renal CS in pre-DM and newly diagnosed Type 2 DM patients compared to subjects with normal glucose metabolism (NGM) and to investigate the usefulness of renal CS in determining the presence of nephropathy in these diseases.

\section{METHODS}

This cross-sectional study included 125 individuals who had three different glucose metabolic states but were not different in terms of age and gender. They were categorised as Group I: subjects with NGM (age $57.4 \pm 6.9$ years; 22 female, 20 male); Group II: patients with pre-DM (age $58.3 \pm 6.9$ years; 28 female, 12 male); and Group III: patients with newly diagnosed Type 2

${ }^{1}$ Department of Internal Medicine, ${ }^{2}$ Department of Radiology, University of Health Sciences, Adana Health Practice and Research Center, Adana, Turkey Correspondence: Dr Ayse Selcan Koc, Department of Radiology, University of Health Sciences, Adana Health Practice and Research Center, Dr Mithat Özsan Bulvarı Kışla Mah, 4522 Sok No. 1, Yüreğir, Adana, Turkey. drayseselcankoc@gmail.com 
DM (age $59.5 \pm 8.5$ years; 24 female, 19 male). Excluded patients had active urinary tract infection, hydronephrosis, kidney stones, renal tumour, polycystic kidney disease, priorly known Type 1 and Type 2 DM, known renal disease, severe heart valve disease, alcohol addiction, abdominal aortic aneurysm and dissection, inflammatory diseases, active thyroid disease, chronic liver disease, cancer, and/or pregnancy. The study was conducted according to the recommendations of the Declaration of Helsinki on biomedical research involving human subjects, and the protocol was approved by the institutional ethics committee. All forms of voluntary consent for all patients were explained in detail and patients were included in the study after receiving written approval.

Detailed anamnesis and physical examinations were performed for the included patients. Subsequently, baseline demographic characteristics of all groups were obtained for age, gender, hypertension, presence of hyperlipidaemia, smoking, coronary artery disease (CAD), peripheral arterial disease (PAD) and stroke history. Pulse rate, systolic blood pressure (SBP) and diastolic blood pressure (DBP) were recorded. Body mass index was calculated by measuring weight and height.

Patients were grouped according to 2016 American Diabetes Association guidelines. ${ }^{(23)}$ Those who had previously been referred to the DM polyclinic based on laboratory results showing unknown glucose metabolism status and who were undiagnosed with pre-DM or DM were divided into three groups according to their glucose metabolism status. These were: (a) accepted as Type 2 DM with glycated haemoglobin (HbA1c) levels $\geq 6.5 \%$, fasting plasma glucose levels $\geq 126 \mathrm{mg} / \mathrm{dL}$ and second-hour plasma glucose levels $75 \mathrm{~g}$ oral glucose tolerance test $(\mathrm{OGTT}) \geq 200 \mathrm{mg} / \mathrm{dL}$; (b) accepted as pre-DM with $\mathrm{HbA} 1 \mathrm{c}$ levels $5.7 \%-6.5 \%$, fasting plasma glucose levels $\geq 100 \mathrm{mg} / \mathrm{dL}$ and $<126 \mathrm{mg} / \mathrm{dL}$ (impaired fasting glucose), and second-hour plasma glucose levels $75 \mathrm{~g}$ OGTT $\geq 140 \mathrm{mg} / \mathrm{dL}$ and $<200 \mathrm{mg} / \mathrm{dL}$ (impaired glucose tolerance); (c) accepted as NGM with $\mathrm{HbA} 1 \mathrm{c}$ levels $<5.7 \%$, fasting plasma glucose levels $<100 \mathrm{mg} / \mathrm{dL}$ and second-hour plasma glucose levels 75 g OGTT $<140$ mg/dL.

Blood samples were taken from an antecubital vein after patients rested for 20 minutes in the supine position. Blood samples were collected in tubes containing ethylenediaminetetraacetic acid. The samples were spun at 3,000 rpm for ten minutes at $0^{\circ} \mathrm{C}$. Fasting blood glucose, blood urea nitrogen, creatinine, total cholesterol, high-density lipoprotein cholesterol, low-density lipoprotein cholesterol and triglyceride levels at the time of admission were measured with the standard automated laboratory method (Abbott Aeroset; Abbott Diagnostics, MN, USA) using appropriate commercial kits (Abbott Aeroset; Abbott Diagnostics, MN, USA). The estimated glomerular filtration rate (eGFR) was calculated using the Modification of Diet in Renal Disease Study Group's formula as follows: eGFR $\left(\mathrm{mL} / \mathrm{min} / 1.73 \mathrm{~m}^{2}\right)=186 \times$ (serum creatinine) $-1.154 \times$ (age) -0.203 (or 0.742 for female patients). ${ }^{(24)}$

$\mathrm{HbA1c}$ measurement was made with an automated chemistry analyser (Abbott Aeroset; Abbott Diagnostics) using appropriate commercial kits (Abbott Aeroset; Abbott Diagnostics). Serum aspartate aminotransferase, alanine aminotransferase, highsensitivity $\mathrm{C}$-reactive protein, $\mathrm{N}$-terminal pro b-type natriuretic peptide (NT-proBNP) and uric acid levels were measured with an automated chemistry analyser (Abbott Aeroset; Abbott Diagnostics) using appropriate commercial kits (Abbott Aeroset; Abbott Diagnostics). Morning urine was used to determine standard albuminuria levels. Albuminuria was determined by calculating the UACR in laboratory results.

Guideline recommendations were used to identify microvascular and macrovascular complications related to Type 2 DM. ${ }^{(23,25-27)}$ Macrovascular complications consisted of the cardiovascular diseases CAD, PAD and stroke. Microvascular complications consisted of nephropathy, retinopathy and neuropathy. The Kidney Disease: Improving Global Outcomes (KDIGO) criterion was used to determine the patient's nephropathy or stage of DKD; DKD was classified into five stages on the basis of eGFR using KDIGO criteria, with eGFR as follows: Stage G1 $\geq 90 \mathrm{~mL} / \mathrm{min} / \mathrm{m}^{2}$, Stage G2 $60-89 \mathrm{~mL} / \mathrm{min} / \mathrm{m}^{2}$, Stage G3 30-59 mL/min/m², Stage G4 15-29 mL/min/m², Stage $\mathrm{G} 5 \leq 15 \mathrm{~mL} / \mathrm{min} / \mathrm{m}^{2}$. For Stages G1 and G2 (eGFR $\left.\geq 60 \mathrm{~mL} / \mathrm{min} / \mathrm{m}^{2}\right)$, the presence of markers of kidney damage (UACR $\geq 30 \mathrm{mg} / \mathrm{g}$ ) is necessary to diagnose DKD. ${ }^{(25)}$ Neuropathy was confirmed by detailed history and evaluation of temperature and vibration sensation. ${ }^{(26)}$ Retinopathy was detected through a comprehensive eye examination. ${ }^{(27)}$

All patients underwent vascular and renal ultrasonography (US) screening using a high-resolution US device (Philips EPIQ 7; Philips Healthcare, Bothell, WA, USA) with a $12 \mathrm{MHz}$ linear and $1-5 \mathrm{MHz}$ high resolution convex probe (Philips Healthcare, Bothell, WA, USA). US examinations were performed after a minimum of six hours of fasting and 20 minutes of rest. The common carotid artery intima-media thickness (IMT) and abdominal aortic IMT were calculated as previously described. ${ }^{(28)}$ Grey-scale B-mode US evaluation was first performed to assess kidney sizes, cortical thickness and parenchyma echogenicity. Kidney length was measured in the coronal plane from the upper pole to the lower pole of the kidney. Renal width was measured from the middle pole and recorded as the distance between the renal hilum and renal capsule. Cortical thickness was recorded as the distance from the medial section of the renal medullary pyramid base to the renal capsule.

SWE evaluation was performed using a 1-5 $\mathrm{MHz}$ convex abdominal probe, the ElastPQ software system and acoustic radiation force impulse technique. All measurements were performed as previously described. ${ }^{(7)}$ For renal US, patients were examined in the left and right lateral decubitus position. The probe was compressed as lightly as possible and placed in a stable position, and the patient was asked not to breathe for a few seconds to minimise the movement of the kidney due to respiration. Measurements were obtained on renal US by placing the region of interest (ROI) on the target (Fig. 1) on the conventional US image, after the target region was determined. The ROI was placed perpendicularly to a vascular-free or cyst-free zone in the renal cortex. The main axis of the ROI was adjusted parallel to the axis of the kidney pyramid (perpendicular to the 


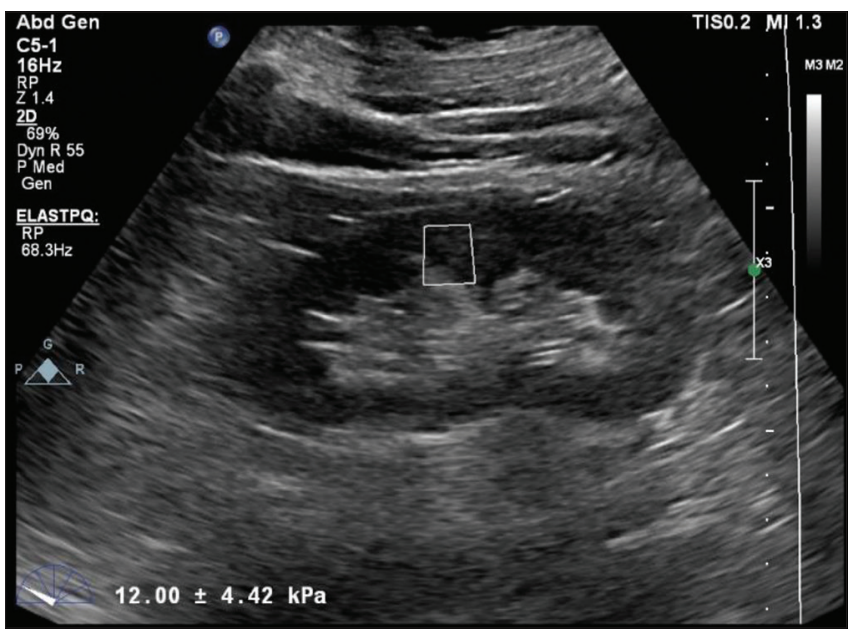

Fig. 1 US image shows cortical stiffness measurement using shear wave elastography in a patient with prediabetes mellitus. Increased shear wave velocity of $12.00 \pm 4.42 \mathrm{kPa}$ is observed.

surface of the kidney). In our study, the maximum ROI target distance was $8 \mathrm{~cm}$ and the ROI fixed box size was $0.5-1 \mathrm{~cm}$. The compression applied was minimised as much as possible during imaging to avoid mechanical pressure to the kidney. The same examination procedure was then repeated for the contralateral kidney. In each case, six valid measurements were obtained for each kidney and the mean value was calculated. If the measurement reliability was low, a result of $0.00 \mathrm{kPa}$ would be displayed. The result was expressed in $\mathrm{kPa}$.

All analyses were performed with IBM SPSS Statistics version 20.0 (IBM Corp, Armonk, NY, USA). Variables were divided into categorical and continuous variables. The normal distribution of continuous variables was assessed using the KolmogorovSmirnov test. Continuous variables in the group were expressed as mean \pm standard deviation. Categorical variables were presented in numbers and percentages. Continuous variables that showed normal distribution were compared using analysis of variance, whereas the Kruskal-Wallis test was used for nonnormally distributed samples. Chi-square test was used to compare categorical variables. Univariate correlation analysis of the parameters associated with renal CS was performed using Pearson's and Spearman's correlation method. Statistically significant parameters were included in the linear regression analysis and the parameters most closely related to renal CS were determined. Receiver operating characteristic curve analysis was performed to determine the patients with nephropathy. Parameters with area under the curve $>0.650$ were determined. From these parameters, limit value determination was done to determine the best sensitivity and specificity in the determination of nephropathy. A p-value $<0.05$ was considered statistically significant.

\section{RESULTS}

The study data was compared by dividing the patients into NGM, pre-DM and Type 2 DM groups according to their glucose metabolism status. All clinical and demographic findings were similar among the groups except for nephropathy frequency, retinopathy frequency, SBP and DBP (Table I). SBP and DBP values were found to be higher in the pre-DM and Type 2 DM groups than the NGM group. The prevalence of nephropathy was found to be $5 \%, 15 \%$ and $33 \%$ in patients with NGM, pre-DM and Type 2 DM, respectively. All laboratory parameters except aspartate aminotransferase and uric acid were different between the groups when the laboratory data was analysed. Glucose, HbA1c, triglyceride, blood urea nitrogen, high-sensitivity C-reactive protein and NT-proBNP levels were significantly higher in patients with Type 2 DM than in the other groups. In the pre-DM and Type 2 DM groups, low-density lipoprotein cholesterol, creatinine and UACR values were found to be higher, while eGFR values were found to be lower compared to the NGM group (Table I).

When the study groups were compared, it was found that all US findings were similar in the pre-DM and Type 2 DM patients. Carotid and aortic IMT, kidney length, and CS values were found to be higher in the pre-DM and Type 2 DM groups than the NGM group (Table II). The renal CS values of the study groups are shown in Fig. 2. Kidney width and cortical thickness values were found to be higher only in Type 2 DM patients compared to NGM group (Table II).

The demographic, clinical, laboratory and US parameters associated with CS in the univariate analysis are summarised in Table III. Linear regression analysis was performed with these CS-related parameters; aortic IMT, kidney length and UACR levels were found to be independently associated with CS. The relationship between CS and UACR and aortic IMT is shown in Fig. 3.

Data on the microvascular and macrovascular organ involvement of the patients with pre-DM and Type $2 \mathrm{DM}$ is shown in Table I. When patients with or without organ involvement were compared, the CS values of patients with and without CAD and/ or PAD, retinopathy and neuropathy were similar (Figs. 4a-C). In patients with nephropathy, CS values were found to be higher than in those without nephropathy $(8.72 \pm 1.67 \mathrm{kPa}$ vs. $10.60 \pm$ $1.74 \mathrm{kPa}, \mathrm{p}=0.001$ and Fig. 4d).

Receiver operating characteristic curve analysis was performed to determine the parameters that best predict the development of nephropathy based on clinical, demographic, laboratory and US data that was different from that in patients with nephropathy (Table IV). The following parameters were found to be significant in the development of nephropathy: UACR, CS, age and aortic IMT (Table IV). When the cut-off value for renal CS was taken as $9.2 \mathrm{kPa}$, renal CS values predicted the possibility of nephropathy with $78.9 \%$ sensitivity and $71.4 \%$ specificity.

\section{DISCUSSION}

The main finding of this study was that renal CS is significantly higher in subjects with Type 2 DM and pre-DM than in those with NGM. Another important finding was the close and independent relationship between renal CS and UACR. We also found increased complications of nephropathy and retinopathy in patients with pre-DM, which was consistent with previous data, as well as in Type 2 DM patients. 
Table I. Clinical, demographic and laboratory findings of the study groups according to glucose metabolism status.

\begin{tabular}{|c|c|c|c|c|}
\hline \multirow[t]{2}{*}{ Variable } & \multicolumn{3}{|c|}{ No. (\%)/mean \pm standard deviation } & \multirow[t]{2}{*}{ p-value } \\
\hline & NGM $(n=42)$ & Pre-DM $(n=40)$ & Type 2 DM (n = 43) & \\
\hline Age (yr) & $57.4 \pm 6.9$ & $58.3 \pm 6.9$ & $59.5 \pm 8.5$ & 0.438 \\
\hline Female gender & $22(52)$ & $28(70)$ & $24(56)$ & 0.236 \\
\hline Hypertension & $7(17)$ & $10(25)$ & $16(37)$ & 0.135 \\
\hline Current smoker & $9(21)$ & $8(20)$ & $8(19)$ & 0.874 \\
\hline Hyperlipidaemia & $7(17)$ & $11(28)$ & $15(35)$ & 0.175 \\
\hline Coronary artery disease & $1(2)$ & $3(8)$ & $10(23)$ & 0.046 \\
\hline Peripheral artery disease & - & $1(3)$ & $5(12)$ & 0.111 \\
\hline Stroke & - & - & $3(7)$ & 0.123 \\
\hline Nephropathy & $2(5)$ & $6(15)$ & $14(33)$ & 0.042 \\
\hline Retinopathy & $1(2.4)$ & $2(5.0)$ & $9(21.0)$ & 0.032 \\
\hline Neuropathy & - & $2(5)$ & $6(14)$ & 0.157 \\
\hline Systolic blood pressure ${ }^{*, t}(\mathrm{mmHg})$ & $124.0 \pm 7.1$ & $130.0 \pm 10.0$ & $131.0 \pm 12.0$ & 0.006 \\
\hline Diastolic blood pressure ${ }^{*, \dagger}(\mathrm{mmHg})$ & $82.0 \pm 5.9$ & $85.0 \pm 8.4$ & $86.0 \pm 8.8$ & 0.016 \\
\hline Heart rate (bpm) & $77 \pm 13$ & $78 \pm 10$ & $80 \pm 12$ & 0.484 \\
\hline Body mass index $\left(\mathrm{kg} / \mathrm{m}^{2}\right)$ & $27.3 \pm 2.1$ & $27.9 \pm 2.4$ & $28.4 \pm 2.2$ & 0.083 \\
\hline Fasting plasma glucose $\mathrm{t}^{\dagger, \ddagger}(\mathrm{mg} / \mathrm{dL})$ & $89.5 \pm 6.0$ & $113.0 \pm 7.4$ & $189.0 \pm 85.0$ & $<0.001$ \\
\hline $\mathrm{HbA} 1 c^{*,+, \neq}(\%)$ & $5.41 \pm 0.23$ & $6.21 \pm 0.58$ & $9.02 \pm 2.22$ & $<0.001$ \\
\hline Total cholesterol ${ }^{*, \neq}(\mathrm{mg} / \mathrm{dL})$ & $135 \pm 17$ & $159 \pm 35$ & $135 \pm 47$ & 0.006 \\
\hline $\operatorname{LDL}_{\text {cholesterol }}^{*, \dagger}(\mathrm{mg} / \mathrm{dL})$ & $97 \pm 17$ & $133 \pm 30$ & $133 \pm 38$ & $<0.001$ \\
\hline $\mathrm{HDL}$ cholesterol ${ }^{\dagger}(\mathrm{mg} / \mathrm{dL})$ & $55.0 \pm 7.6$ & $52.0 \pm 14.0$ & $45.0 \pm 15.0$ & 0.006 \\
\hline Triglycerides $^{+, \neq}(\mathrm{mg} / \mathrm{dL})$ & $91 \pm 32$ & $128 \pm 48$ & $233 \pm 165$ & $<0.001$ \\
\hline Aspartate aminotransferase (IU/L) & $19.1 \pm 3.1$ & $19.9 \pm 5.4$ & $21.8 \pm 7.9$ & 0.089 \\
\hline Alanine aminotransferase ${ }^{\dagger}$ (IU/L) & $14.5 \pm 2.7$ & $17.8 \pm 7.9$ & $21.1 \pm 11.0$ & 0.001 \\
\hline Blood urea nitrogen ${ }^{t, \neq}(\mathrm{mg} / \mathrm{dL})$ & $26.2 \pm 4.7$ & $23.6 \pm 6.3$ & $33.6 \pm 13.0$ & $<0.001$ \\
\hline Creatinine $^{*, \dagger}(\mathrm{mg} / \mathrm{dL})$ & $0.54 \pm 0.06$ & $0.68 \pm 0.15$ & $0.71 \pm 0.19$ & $<0.001$ \\
\hline $\mathrm{eGFR}^{*, \dagger}\left(\mathrm{mL} / \mathrm{min} / 1.73 \mathrm{~m}^{2}\right)$ & $138 \pm 18$ & $110 \pm 18$ & $106 \pm 25$ & $<0.001$ \\
\hline Uric acid (mg/dL) & $4.41 \pm 0.69$ & $4.70 \pm 0.68$ & $5.03 \pm 2.17$ & 0.158 \\
\hline $\mathrm{hs}-\mathrm{CRP}^{\mathrm{t}, \mathrm{f}}(\mathrm{mg} / \mathrm{dL})$ & $0.21 \pm 0.11$ & $0.43 \pm 0.18$ & $0.89 \pm 0.94$ & $<0.001$ \\
\hline NT-proBNP*,t,‡ $(\mathrm{pg} / \mathrm{mL})$ & $78 \pm 21$ & $63 \pm 20$ & $301 \pm 240$ & $<0.001$ \\
\hline $\mathrm{UACR}^{*, \dagger}(\mathrm{mg} / \mathrm{g})$ & $11.1 \pm 5.3$ & $21.6 \pm 12.0$ & $59.6 \pm 111.0$ & 0.002 \\
\hline
\end{tabular}

${ }^{*} \mathrm{p}<0.05$ between NGM and pre-DM groups, +NGM and Type 2 DM groups, fpre-DM and Type 2 DM groups. DM: diabetes mellitus; eGFR: estimated glomerular filtration rate; HbA1c: glycated haemoglobin; HDL: high-density lipoprotein; hs-CRP: high-sensitivity C-reactive protein; LDL: low-density lipoprotein; NGM: normal glucose metabolism; NT-proBNP: N-terminal pro b-type natriuretic peptide; UACR: urinary albumin-creatinine ratio

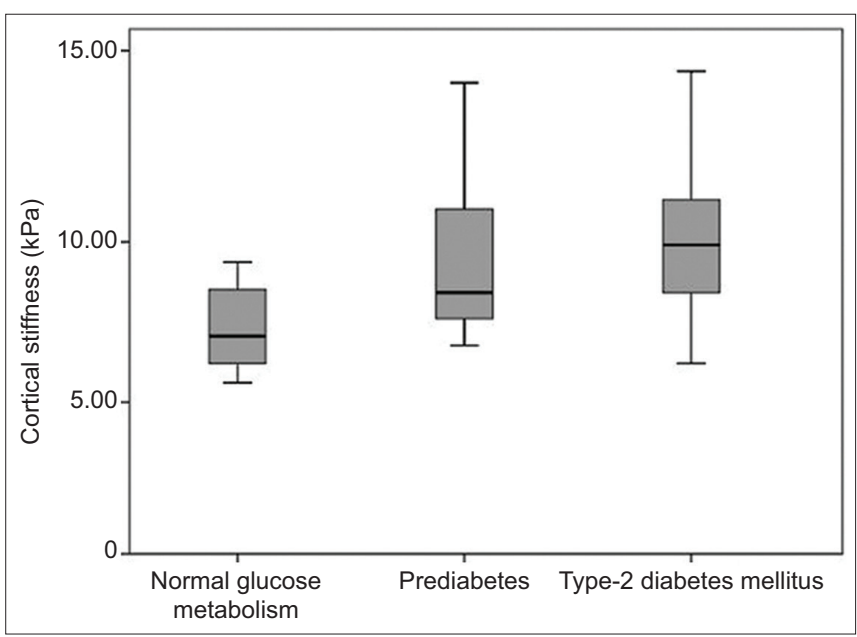

Fig. 2 Box plot shows renal cortical stiffness values according to the glucose metabolism status of the study groups.
In patients with pre-DM, both impaired glucose tolerance and impaired fasting glucose are associated with a cardiovascular event. ${ }^{(1)}$ Undiagnosed myocardial infarction is more prevalent in patients with pre-DM than in those with NGM $(3.5 \%$ vs. $1.4 \%) .{ }^{(9)}$ Furthermore, a meta-analysis of 17 trials showed increased CAD, PAD and stroke frequency. ${ }^{(1,10,11)}$ In our study, $10 \%$ of patients with pre-DM had macrovascular complications.

The development of retinopathy in Type $2 \mathrm{DM}$ is well known: there is a risk of retinopathy in the pre-DM stage, when it is more common than in NGM patients with a prevalence of about $8 \% .^{(2,4)}$ Retinopathy prevalence was reported to be $6.7 \%$ and $8.2 \%$ in the other two studies. ${ }^{(3,4)}$ In our study, the prevalence of retinopathy in NGM, pre-DM and Type 2 DM patients was significantly different at $2.4 \%, 5.0 \%$ and $21.0 \%$, respectively. While there is no clear data on neuropathy in those with pre-DM, it has been reported that in a study of prediabetic patients, the frequency 
Table II. Vascular and renal ultrasonography findings of the study groups according to glucose metabolism status.

\begin{tabular}{|c|c|c|c|c|}
\hline \multirow[t]{2}{*}{ Variable } & \multicolumn{3}{|c|}{ Mean \pm standard deviation } & \multirow[t]{2}{*}{ p-value } \\
\hline & NGM $(n=42)$ & Pre-DM $(n=40)$ & Type 2 DM $(n=43)$ & \\
\hline Carotid IMT ${ }^{*, \dagger}(\mathrm{mm})$ & $0.61 \pm 0.09$ & $0.74 \pm 0.10$ & $0.80 \pm 0.13$ & $<0.001$ \\
\hline Aortic IMT ${ }^{*, t}(\mathrm{~mm})$ & $1.17 \pm 0.15$ & $1.63 \pm 0.43$ & $1.83 \pm 0.42$ & $<0.001$ \\
\hline Kidney length ${ }^{*,+}(\mathrm{cm})$ & $98 \pm 3.4$ & $101 \pm 5.7$ & $105 \pm 12$ & $<0.001$ \\
\hline Kidney width ${ }^{+}(\mathrm{cm})$ & $44 \pm 4.9$ & $45 \pm 5.9$ & $49 \pm 8.1$ & 0.004 \\
\hline Cortical thickness $^{\dagger}(\mathrm{mm})$ & $12.1 \pm 1.2$ & $12.5 \pm 1.7$ & $13.9 \pm 2.4$ & $<0.001$ \\
\hline Renal echogenicity ${ }^{\ddagger}$ & & & & 0.002 \\
\hline 0 & 38 & 32 & 28 & \\
\hline 1 & 3 & 7 & 12 & \\
\hline II & 1 & 1 & 4 & \\
\hline III & 0 & 0 & 0 & \\
\hline ROI depth (cm) & $5.47 \pm 1.5$ & $5.70 \pm 1.2$ & $5.19 \pm 1.3$ & 0.694 \\
\hline Cortical stiffness ${ }^{*, t}(\mathrm{kPa})$ & $6.93 \pm 0.96$ & $8.58 \pm 1.93$ & $9.27 \pm 1.76$ & $<0.001$ \\
\hline
\end{tabular}

${ }^{*} \mathrm{p}<0.05$ between NGM and pre-DM groups, + NGM and Type 2 DM groups. $\neq$ Data presented as number of patients. DM: diabetes mellitus; IMT: intima-media thickness; NGM: normal glucose metabolism; ROI: region of interest
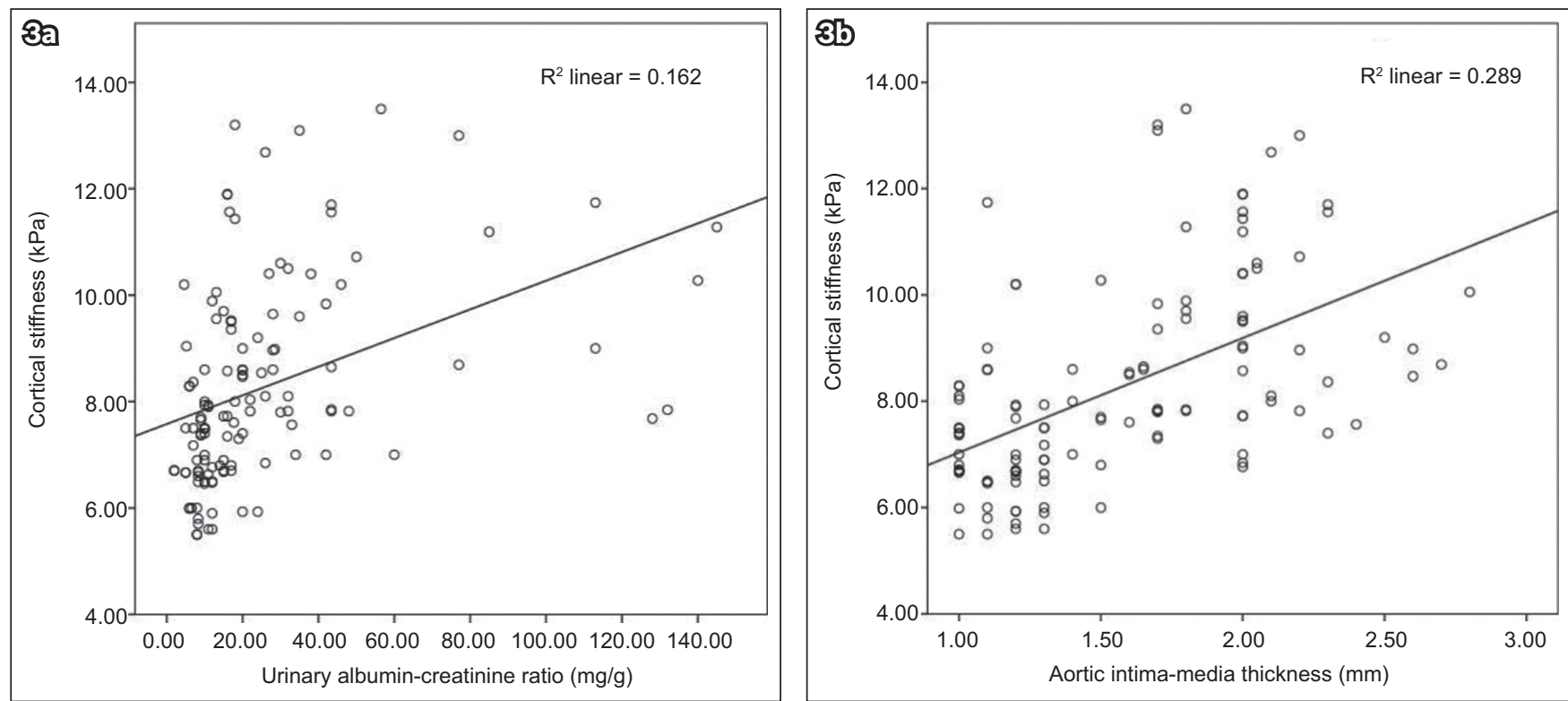

Fig. 3 Scatter plots show a significant correlation between (a) renal cortical thickness and urinary albumin-creatinine ratio; and (b) renal cortical thickness and aortic intima-media thickness.

of autonomic neuropathy was $8 \%-9.8 \%{ }^{(8)}$ In our study, there was no neuropathy in those with NGM, but DM patients had an increased frequency of neuropathy compared to those with pre-DM; however, the difference was not statistically significant (14\% vs. $5 \%$, p = 0.157). 20\%-30\% of patients with Type 2 DM develop nephropathy in the long term, while the most important problem in DM patients is DKD. ${ }^{(19)}$ Individuals diagnosed with pre-DM have a higher incidence of nephropathy than those with NGM and the most common form of microvascular organ involvement among them is nephropathy. ${ }^{(1)}$ The National Health and Nutrition Examination Survey's data analysis found that the prevalence of chronic renal disease was $11.8 \%, 17.1 \%$ and $24.2 \%$ in non-DM, pre-DM and newly diagnosed DM patients, respectively. ${ }^{(5)}$ In our study, the prevalence of nephropathy in NGM, pre-DM and DM patients was 5\%, 15.0\% and 33.0\%, respectively.
In a study conducted on a large group of patients in Korea, patients in the pre-DM stage had more proteinuria than normal individuals $(6.6 \%$ vs. $3.6 \%) .{ }^{(7)}$ A study conducted by Metcalf et $\mathrm{a}^{(6)}$ once reported that nephropathy associated with microalbuminuria was more prevalent in the stage of pre-DM than in NGM (16.1\% vs. $4 \%$ ). This rate of nephropathy is very close to that of our study. Different parameters have been used for detecting the development of nephropathy, with the most commonly used test investigating the presence of albuminuria in spot or 24-hour urine. In our study, a KDIGO criterion that includes albuminuria was used for detection of nephropathy. ${ }^{(25)}$ Previous studies have reported that albuminuria is significantly higher in patients with pre-DM than in those with NGM. ${ }^{(29-32)}$

DM is known to cause progressive renal disease with glomerular hyperfiltration. A study conducted by Melsom et al ${ }^{(12)}$ in patients with pre-DM and another study performed with 


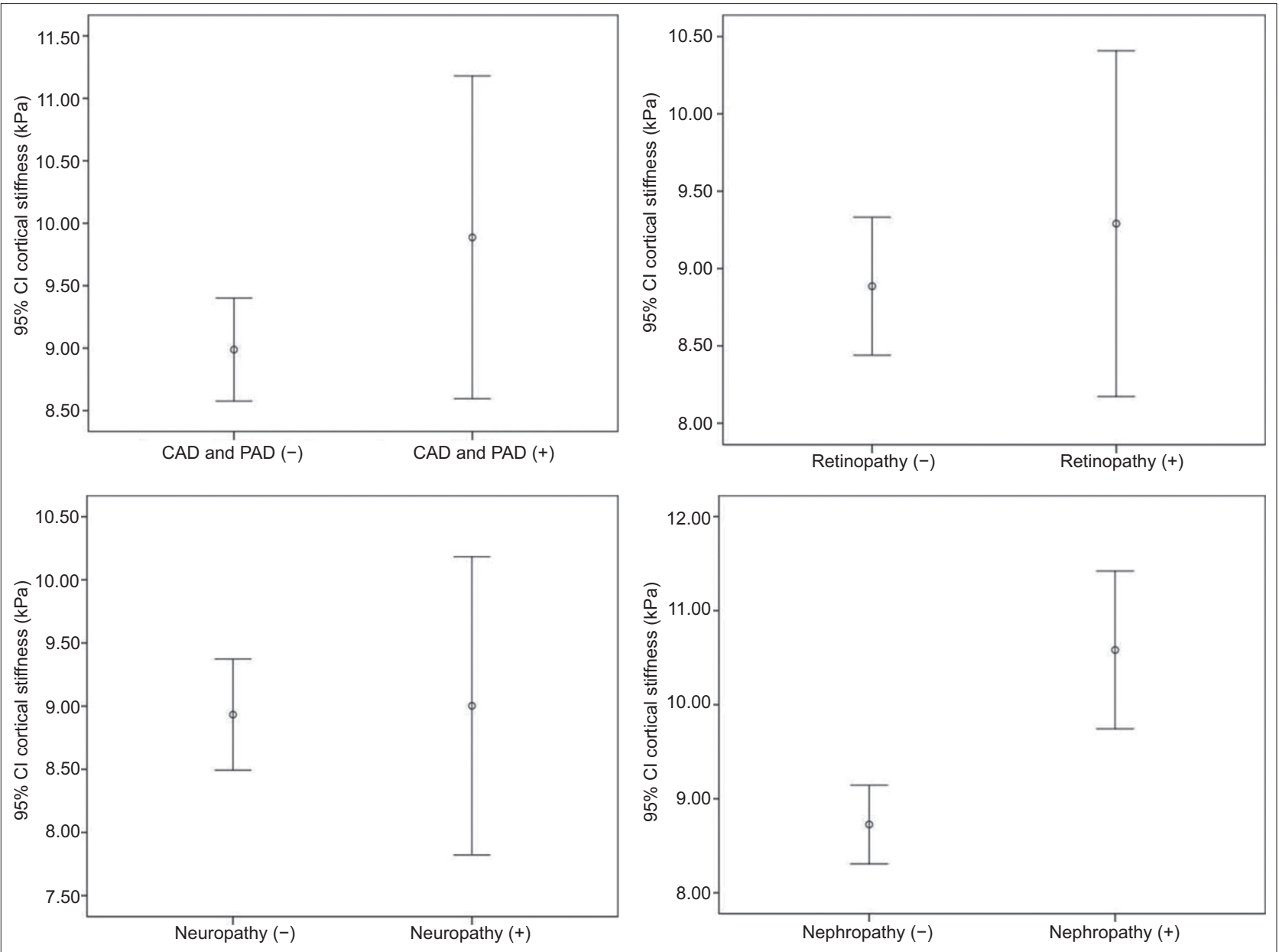

Fig. 4 Box plots show renal cortical stiffness values in patients with microvascular and macrovascular complications. CAD: coronary artery disease; Cl: confidence interval; PAD: peripheral artery disease

Table III. Parameters associated with renal cortical stiffness and linear regression analysis for parameters significantly correlated with renal cortical stiffness.

\begin{tabular}{|c|c|c|c|c|}
\hline \multirow[t]{2}{*}{ Variable } & \multicolumn{2}{|c|}{$\begin{array}{c}\text { Univariate } \\
\text { analysis }\end{array}$} & \multicolumn{2}{|c|}{$\begin{array}{c}\text { Multivariate } \\
\text { analysis }\end{array}$} \\
\hline & p-value & $\mathbf{r}$ & p-value & $\boldsymbol{\beta}$ \\
\hline Age (yr) & 0.018 & 0.211 & 0.979 & 0.143 \\
\hline Creatinine (mg/dL) & 0.019 & 0.211 & 0.813 & 0.078 \\
\hline $\begin{array}{l}\text { Fasting plasma glucose } \\
(\mathrm{mg} / \mathrm{dL})\end{array}$ & $<0.001$ & 0.413 & 0.521 & 0.056 \\
\hline $\mathrm{HbA1c}(\%)$ & $<0.001$ & 0.477 & 0.515 & 0.105 \\
\hline eGFR (mL/min/1.73 $\left.\mathrm{m}^{2}\right)$ & $<0.001$ & -0.318 & 0.730 & -0.091 \\
\hline Creatinine (mg/dL) & 0.002 & 0.273 & 0.630 & 0.102 \\
\hline Carotid IMT (mm) & $<0.001$ & 0.427 & 0.770 & 0.148 \\
\hline Aortic IMT (mm) & $<0.001$ & 0.537 & $<0.001$ & 0.443 \\
\hline Kidney length (cm) & $<0.001$ & 0.359 & 0.005 & 0.219 \\
\hline Kidney width $(\mathrm{cm})$ & 0.022 & 0.207 & 0.613 & 0.195 \\
\hline Renal echogenicity & 0.001 & 0.385 & 0.713 & 0.099 \\
\hline Cortical thickness (mm) & 0.002 & 0.385 & 0.778 & 0.058 \\
\hline UACR (mg/g) & 0.001 & 0.288 & 0.002 & 0.233 \\
\hline
\end{tabular}

Adjusted $\mathrm{R}^{2}=0.406$ in multivariate analysis. eGFR: estimated glomerular filtration rate; HbA1c: glycated haemoglobin; IMT: intima-media thickness; UACR: urinary albumin-creatinine ratio patients in the early stages of DM showed that hyperglycaemia and glomerular hyperfiltration were associated with albuminuria development. ${ }^{(18)}$ The albuminuria mechanism that occurs in the pre-DM stage can be explained by the presence of glycation and thickening in glomerular basal membrane proteins caused by hyperglycaemia, increase in intraglomerular pressure, the loss of selective permeability of the glomerular membrane, glomerular hyperperfusion and hyperfiltration. ${ }^{(33)}$ Our study also found that UACR-detected albuminuria was significantly higher in both the DM and pre-DM groups than in those with NGM. In addition, there was a close and independent relationship between UACR and renal CS. This association is thought to be due to the physiopathologic changes causing albuminuria in the early phase of impaired glucose metabolism and can be shown objectively through renal elastography. With early detection of increased CS, albuminuria or DKD progression in pre-DM or early DM can be reduced or prevented with diet, exercise and, if necessary, medical treatment.

Conventional renal US is another examination to detect the presence of nephropathy or differential diagnosis in DM or prediabetic patients. Due to the hyperfiltration of DM and preDM, morphological changes such as increased echogenicity and increased renal size may be seen on conventional US, but it does 
Table IV. Receiver operating characteristic curve analysis for predicting the presence of nephropathy.

\begin{tabular}{|llllll|}
\hline Variable & AUROC curve & p-value & Cut-off & Sensitivity & Specificity \\
\hline UACR $(\mathrm{mg} / \mathrm{g})$ & $0.877(0.800-0.953)$ & $<0.001$ & 30 & $94.4 \%$ & $82.3 \%$ \\
\hline Cortical stiffness $(\mathrm{kPa})$ & $0.792(0.687-0.897)$ & $<0.001$ & 9.2 & $78.9 \%$ & $71.4 \%$ \\
\hline Age $(\mathrm{yr})$ & $0.656(0.518-0.793)$ & 0.045 & 60 & $72.3 \%$ & $66.1 \%$ \\
\hline Aortic IMT $(\mathrm{mm})$ & $0.655(0.516-0.783)$ & 0.047 & 1.8 & $72.2 \%$ & $60.7 \%$ \\
\hline
\end{tabular}

AUROC: area under receiver operating characteristic; IMT: intima-media thickness; UACR: urinary albumin-creatinine ratio

not provide specific information for the detection of nephropathy in newly diagnosed Type 2 DM and prediabetic cases. CS values obtained in SWE tests have been more popular recently. ${ }^{(21)}$ SWE is a cost-effective, reliable and noninvasive US test that can be used to determine tissue elasticity. ${ }^{(34)}$ Renal parenchymal fibrosis is the most important sign of kidney disease, causing changes in mechanical features of kidneys that can be measured objectively with SWE. ${ }^{(21)}$ Increases in renal CS, as determined by SWE, are known to occur in patients with Type 2 DM. ${ }^{(18,20,21)}$ However, to our knowledge, there is no information regarding CS changes in patients with pre-DM in the literature. Studies revealed that changes at the cellular level occur prior to the diagnosis of abnormalities associated with nephropathy. ${ }^{(19)}$ The limitations of SWE are its inconsistent availability in clinics and the absence of standardised normal values of CS for DM and prediabetic patients.

In the present study, a $9.2 \mathrm{kPa}$ cut-off value for CS could determine the possibility of nephropathy with $78.9 \%$ sensitivity and $71.4 \%$ specificity. However, our data was not comparable with that of other studies because their patients with pre-DM or newly diagnosed Type 2 DM had no prior CS studies with renal elastography. Development of DKD is still observed in DM patients and is the main risk factor for cardiovascular diseases. For this reason, it is important to diagnose DM patients in stages of preDM and to diagnose pre-DM before the presence of microvascular organ involvement. Renal CS is higher in those with impaired glucose metabolism than with NGM. In the subgroup analysis, CS increased in DM patients compared to the patients with pre-DM, but the difference was not statistically significant, suggesting that the increase in renal CS occurred during the early stage of impaired glucose metabolism. Our study also showed a significant association between renal CS and UACR, which is the first time this relationship has been shown, to the best of our knowledge. This finding should be confirmed with further studies involving many more patients, because renal CS is an objective, stable parameter and can be used in the early detection of microvascular complications, preferably before the onset of newly acquired albuminuria.

The present study had some important limitations. First, the sample size was relatively small and the study was conducted in a single centre. A follow-up multicentre study with more patients is required. Second, our study was cross-sectional and patients with newly diagnosed Type 2 DM were included, but data on the disease duration since first diagnosis was not available, and thus we could not find out whether DM duration affected the results. Third, some patients were excluded from the study due to the technical limitations of our equipment. For example, we had to exclude patients with renal cortical parenchymal thickness $<1 \mathrm{~cm}$ and those with deeply located kidneys $(>8 \mathrm{~cm}$ from the skin surface) because the ROI target distance of our US equipment was a maximum of $8 \mathrm{~cm}$ and the ROI constant box size was $0.5-1 \mathrm{~cm}$.

In conclusion, renal CS values in patients with newly diagnosed Type 2 DM and pre-DM were significantly higher than that in those with NGM, and were closely related to nephropathy development. There was also a very close relationship between renal CS and UACR. Our study findings suggest that increased CS as seen on renal elastography may predate or predetermine the development of nephropathy, which is, in turn, the most frequent microvascular complication in patients with impaired glucose metabolism. Hence, we conclude that routine measurement of renal CS with renal elastography in patients with pre-DM and DM may be useful to identify patients at risk for DKD even before microvascular complications are fully manifested in these patients. It is important to diagnose DM patients in pre-DM stages and to diagnose pre-DM before the presence of microvascular organ involvement. For this purpose, besides the periodic use of microalbuminuria, subsequent measurements of CS using renal elastography may be important and can be used periodically at patient follow-up.

\section{REFERENCES}

1. Kumar R, Nandhini LP, Kamalanathan S, Sahoo J, Vivekanadan M. Evidence for current diagnostic criteria of diabetes mellitus. World J Diabetes 2016; 7:396-405.

2. Diabetes Prevention Program Research Group. The prevalence of retinopathy in impaired glucose tolerance and recent-onset diabetes in the Diabetes Prevention Program. Diabet Med 2007; 24:137-44.

3. Tapp RJ, Shaw JE, Harper CA, et al; AusDiab Study Group. The prevalence of and factors associated with diabetic retinopathy in the Australian population. Diabetes Care 2003; 26:1731-7.

4. Lamparter J, Raum P, Pfeiffer N, et al. Prevalence and associations of diabetic retinopathy in a large cohort of prediabetic subjects: the Gutenberg Health Study. J Diabetes Complications 2014; 28:482-7.

5. Plantinga LC, Crews DC, Coresh J, et al; CDC CKD Surveillance Team. Prevalence of chronic kidney disease in US adults with undiagnosed diabetes or pre-DM. Clin J Am Soc Nephrol 2010; 5:673-82.

6. Metcalf PA, Baker JR, Scragg RK, et al. Microalbuminuria in a middle-aged workforce. Effect of hyperglycemia and ethnicity. Diabetes Care 1993; 16:1485-93.

7. Kim $\mathrm{CH}, \mathrm{Kim} \mathrm{KJ}, \mathrm{Kim} \mathrm{BY}$, et al. Pre-DM is not independently associated with microalbuminuria in Korean general population: the Korea National Health and Nutrition Examination Survey 2011-2012 (KNHANES V-2,3). Diabetes Res Clin Pract 2014; 106:e18-21.

8. Kannan MA, Sarva S, Kandadai RM, et al. Prevalence of neuropathy in patients with impaired glucose tolerance using various electrophysiological tests. Neurol India 2014; 62:656-61.

9. Barr EL, Zimmet PZ, Welborn TA, et al. Risk of cardiovascular and all-cause mortality in individuals with diabetes mellitus, impaired fasting glucose, and impaired glucose tolerance: the Australian Diabetes, Obesity, and Lifestyle Study (AusDiab). Circulation 2007; 116:151-7.

10. Xu T, Liu W, Cai X, et al. Risk of coronary heart disease in different criterion of impaired fasting glucose: a meta-analysis. Medicine (Baltimore) 2015; 94:e1740.

11. Lee M, Saver JL, Hong KS, et al. Effect of pre-diabetes on future risk of stroke: meta-analysis. BMJ 2012; 344:e3564.

12. Melsom T, Schei J, Stefansson VT, et al. Pre-DM and risk of glomerular hyperfiltration and albuminuria in the general nondiabetic population: a 
prospective cohort study. Am J Kidney Dis 2016; 67:841-50.

13. Ligthart S, van Herpt TT, Leening MJ, et al. Lifetime risk of developing impaired glucose metabolism and eventual progression from pre-DM to type 2 diabetes: a prospective cohort study. Lancet Diabetes Endocrinol 2016; 4:44-51.

14. Guariguata L, Whiting DR, Hambleton I, et al. Global estimates of diabetes prevalence for 2013 and projections for 2035. Diabetes Res Clin Pract 2014; 103:137-49.

15. Echouffo-Tcheugui JB, Narayan KM, Weisman D, Golden SH, Jaar BG. Association between pre-DM and risk of chronic kidney disease: a systematic review and meta-analysis. Diabet Med 2016; 33:1615-24.

16. Olivarius Nde F, Andreasen AH, Keiding N, Mogensen CE. Epidemiology of renal involvement in newly-diagnosed middle-aged and elderly diabetic patients. Cross-sectional data from the population-based study "Diabetes Care in General Practice", Denmark. Diabetologia 1993; 36:1007-16.

17. Davis TM, Stratton IM, Fox CJ, Holman RR, Turner RC. U.K. Prospective Diabetes Study 22. Effect of age at diagnosis on diabetic tissue damage during the first 6 years of NIDDM. Diabetes Care 1997; 20:1435-41.

18. De Nicola L, Gabbai F, Liberti ME, et al. Sodium/glucose cotransporter 2 inhibitors and prevention of diabetic nephropathy: targeting the renal tubule in diabetes. Am J Kidney Dis 2014; 64:16-24

19. Shahbazian H, Rezaii I. Diabetic kidney disease; review of the current knowledge. J Renal Inj Prev 2013; 2:73-80.

20. Goya C, Kilinc F, Hamidi C, et al. Acoustic radiation force impulse imaging for evaluation of renal parenchyma elasticity in diabetic nephropathy. AJR Am J Roentgenol 2015; 204:324-9.

21. Hassan K, Loberant N, Abbas N, et al. Shear wave elastography imaging for assessing the chronic pathologic changes in advanced diabetic kidney disease. Ther Clin Risk Manag 2016; 12:1615-22.

22. $\mathrm{Yu} \mathrm{N}$, Zhang $\mathrm{Y}, \mathrm{Xu} \mathrm{Y}$. Value of virtual touch tissue quantification in stages of diabetic kidney disease. J Ultrasound Med 2014; 33:787-92.

23. American Diabetes Association. 2. Classification and diagnosis of diabetes. Diabetes Care 2016; 39 Suppl 1:S13-22.

24. Levey AS, Bosch JP, Lewis JB, et al. A more accurate method to estimate glomerular filtration rate from serum creatinine: a new prediction equation.
Modification of Diet in Renal Disease Study Group. Ann Intern Med 1999; 130:461-70.

25. KDIGO 2012 Clinical Practice Guideline for the Evaluation and Management of Chronic Kidney Disease. Kidney Int Suppl 2013; 3:1-150. Available at: https:// kdigo.org/wp-content/uploads/2017/02/KDIGO_2012_CKD_GL.pdf. Accessed May 21, 2019

26. Hendrick AM, Gibson MV, Kulshreshtha A. Diabetic retinopathy. Prim Care 2015; 42:451-64

27. Russell JW, Zilliox LA. Diabetic neuropathies. Continuum (Minneap Minn) $2014 ; 20: 1226-40$

28. Koc AS, Gorgulu FF, Donmez Y, Icen YK. There is a significant relationship between morning blood pressure surge and increased abdominal aortic intimamedia thickness in hypertensive patients. J Med Ultrason (2001) 2018; 45:597603.

29. Bahar A, Makhlough A, Yousefi A, Kashi Z, Abediankenari S. Correlation between pre-DM conditions and microalbuminuria. Nephrourol Mon 2013; 5:741-4.

30. Franciosi M, Pellegrini F, Sacco M, et al; IGLOO (Impaired Glucose tolerance, and Long-term Outcomes Observational Study) Study Group. Identifying patients at risk for microalbuminuria via interaction of the components of the metabolic syndrome: a cross-sectional analytic study. Clin J Am Soc Nephrol 2007; 2:984-91.

31. Tapp RJ, Shaw JE, Zimmet PZ, et al. Albuminuria is evident in the early stages of diabetes onset: results from the Australian Diabetes, Obesity, and Lifestyle Study (AusDiab). Am J Kidney Dis 2004; 44:792-8.

32. Wang $\mathrm{XL}$, Lu JM, Pan CY, Tian H, Li CL. A comparison of urinary albumin excretion rate and microalbuminuria in various glucose tolerance subjects. Diabet Med 2005; 22:332-5.

33. Tarsio JF, Reger LA, Furcht LT. Molecular mechanisms in basement membrane complications of diabetes. Alterations in heparin, laminin, and type IV collagen association. Diabetes 1988; 37:532-9.

34. Zaffanello M, Piacentini G, Bruno C, Brugnara M, Fanos V. Renal elasticity quantification by acoustic radiation force impulse applied to the evaluation of kidney diseases: a review. J Investig Med 2015; 63:605-12. 